\title{
Intracranial aneurysms Two options are better than only one
}

\author{
Paulo Puglia Jr.
}

In this issue, Arquivos de NeuroPsiquiatria publishes three articles concerning the therapeutics of intracranial aneurysms (IA). These papers have many positive aspects, especially because they bring the debate on this topic to an important journal that is read by many neurologists and neurosurgeons. Although they are very different, our position on the long path towards definitive answers may be shown by highlighting their central questions.

Permeating the three articles is the fact that there are currently two different treatments for intracranial aneurysms: microsurgical clipping (MC) and endovascular coiling (EC). Since EC was introduced more recently, it is natural that it has to be proven to be at least as efficient and safe as MC, for it to be accepted.

However, if the first treatment that existed was perfect, would anybody look for another one? When it was first introduced, EC was applied only to patients who were considered unfit for open surgery. This made it possible to offer some form of treatment for patients without any other option. The results were fairly good, and after years of improvements and research, EC became an option even for treating aneurysms that could be resolved by means of $\mathrm{MC}^{1,2}$. Advantages for both types of treatment have been demonstrated, and great controversy persists with regard to choosing between them for a particular case. In general terms, EC is preferred for aneurysms located in poorly accessible sites, for ruptured aneurysms with severe clinical and neurological con- ditions and for older patients, with the advantage of prompt postoperative recuperation. The main limitation is aneurysm geometry: cases with a wide neck or large neck/sac ratio, especially if originating in a bifurcation, give rise to complications because of coil protrusion towards the normal vessel. There is also a disconcerting risk of recurrence (about 20\%) and need for retreatment $(10 \%)^{3}$. Balloon remodeling and stent devices minimize these limitations, but make the procedure more risky and expensive. Other features can turn EC into a difficult task, like vessel tortuosity and very small sized sacs. Craniotomy and $\mathrm{MC}$ work best for anterior circulation lesions, independently of the neck/sac ratio and location at bifurcations, and for young patients. There are aneurysms with good prognoses from both treatments, and the contrary. Giant, partially thrombosed, wide-necked aneurysms of the posterior circulation are associated with poor results for both techniques.

Taking the theme of showing that EC is a good treatment, the article by Abud et al. reports on a series of selected patients who were treated with four different types of EC. They showed that interventional physicians learned to choose the cases in which their techniques would do well. What we cannot know is what proportion of patients presenting to hospital could be treated this way. In the ISAT study, only one quarter of the patients investigated were considered fit for $\mathrm{EC}$ and $\mathrm{MC}$ concomitantly. Thus, it is reasonable to assume that the patients treated represent only a proportion of those considered
Correspondence

uglia Jr.

Rua Inácio Pereira da Rocha 486 05432-011 São Paulo SP - Brasil

E-mail: ppuglia@uol.com.br
ANEURISMAS INTRACRANIANOS: DUAS OPÇÕES SÃO MELHORES OUE UMA

${ }^{1} \mathrm{MD}$, PhD. Department of Radiology, University of São Paulo Medical School, São Paulo SP, Brazil. 
for EC. This reveals a limitation of the method, even with its four methods. It is remarkable, however, that "most" of the cases were considered unfit for $\mathrm{MC}$, at least in the judgment of the neurosurgeons at that particular center. In these cases, EC was a treatment that was complementary to $\mathrm{MC}$.

Concurrency created by new therapeutic methods has aided in searching for fragilities within surgical methods and in solving them. Along these lines, the article by Pereira-Filho et al. investigates possible hazards associated with $\mathrm{MC}$, and their concern is admirable. If a hazard exists, knowing about it is the first step towards avoiding it. Perhaps this worry would not appear if there was no competing therapeutic option. Since embolization is carried out without parenchymal manipulation and with a low risk of perforating lesions or ligation, it is believed to be less prone to cause neuropsychological damage, especially in operations involving the anterior communicating region, the carotid bifurcation and the basilar apex ${ }^{4}$. Only a quarter of the aneurysms investigated were located in these regions, and, as mentioned by the authors, other more detailed tests would have shown more subtle alterations. Nevertheless, the intention to search for postoperative abnormalities is positive in itself.

In a case report, Christoph et al. raise the question of choice when both EC and MC are considered feasible. This question is very complex, involving individual judgment, local particularities and patient wishes. Every judgment is subject to error, despite the intention and desire of making the correct choice. When interventional physicians consider that EC is feasible in cases of aneurysm but then discover that it is not feasible, there is still a chance to stop the procedure before a coil has yet been detached. Subsequently, a rescue procedure like stent placement or even surgery may be necessary, thus adding a risk of morbidity. This case also illustrates the need for collaboration between the neurosurgical and intervention teams. However, the risks of over-indication must be remembered and, even though patients' opinions are important, they must come after the technical possibilities have been determined.

In conclusion, the introduction of a new treatment alongside an established one will be marked by controversy, and is a gradual process, taking many years. Nonetheless, this cannot be regarded as anything other than an integral part of progress. The old and new techniques are sometimes complementary and sometimes in competition, but patients are always favored.

\section{REFERENCES}

1. Molyneux A, Kerr R, Stratton I, et al. International subarachnoid aneurysm trial (ISAT) of neurosurgical clipping versus endovascular coiling in 2143 patients with ruptured intracranial aneurysms: a randomised trial. Lancet 2002;360:1267-1274.

2. Meyers PM, Schumacher HC, Higashida RT, et al. American Heart Association. Indications for the performance of intracranial endovascular neurointerventional procedures: a scientific statement from the American Heart Association Council on Cardiovascular Radiology and Intervention, Stroke Council, Council on Cardiovascular Surgery and Anesthesia, Interdisciplinary Council on Peripheral Vascular Disease, and Interdisciplinary Council on Quality of Care and Outcomes Research. Circulation 2009;119:2235-2249.

3. Ferns SP, Sprengers ME, van Rooij WJ, et al. Coiling of intracranial aneurysms: a systematic review on initial occlusion and reopening and retreatment rates. Stroke 2009;40:523-529.

4. Scott RB, Eccles F, Molyneux AJ, Kerr RS, Rothwell PM, Carpenter K. Improved cognitive outcomes with endovascular coiling of ruptured intracranial aneurysms: neuropsychological outcomes from the international subarachnoid aneurysm trial (ISAT). Stroke 2010;41:1743-1747. 